\title{
Intramolecular Charge Transfer with N,N-Dialkyl-4-(Trifluoromethyl)anilines and 4-(Dimethylamino)benzonitrile in Polar Solvents. Investigation of the Excitation Wavelength Dependence of the Reaction Pathway
}

\author{
V.A. GalievskY* And K.A. Zachariasse ${ }^{\dagger}$ \\ Max-Planck-Institut für biophysikalische Chemie, \\ Spektroskopie und Photochemische Kinetik \\ 37070 Göttingen, Germany
}

Klaas Zachariasse dedicates this paper to the dear memory of his friend Jurek Prochorow

\begin{abstract}
In the series of N,N-di-n-alkyl-4-(trifluoromethyl)anilines (DXCF3), with $\mathrm{X}=\mathrm{M}$ (methyl), E (ethyl), Pr ( $n$-propyl) and Pe ( $n$-pentyl), an intramolecular charge transfer (ICT) reaction takes place in the polar solvent acetonitrile (MeCN). Such a reaction does not occur in the less polar solvents $n$-hexane and diethyl ether, in which a single fluorescence band from the locally excited (LE) state is observed. For DMCF3 in MeCN at $25^{\circ} \mathrm{C}$, the intramolecular charge transfer reaction efficiency is much smaller than for 4-(dimethylamino)benzonitrile (DMABN) in this solvent, as seen from the ICT/LE fluorescence quantum yield ratio $\Phi^{\prime}(\mathrm{ICT}) / \Phi(\mathrm{LE}): 0.06$ for DMCF3 and 39.5 for DMABN. As predicted by the planar ICT (PICT) model, this difference is caused by the considerably larger energy gap $\Delta E\left(S_{1}, S_{2}\right)$ of DMCF3 $\left(5750 \mathrm{~cm}^{-1}\right)$ as compared with that of DMABN $\left(3130 \mathrm{~cm}^{-1}\right)$. When the alkyl chain length of DXCF3 becomes larger, $\Phi^{\prime}(\mathrm{ICT}) / \Phi(\mathrm{LE})$ in $\mathrm{MeCN}$ at $25^{\circ} \mathrm{C}$ increases, from 0.06 for DMCF3 to 0.64 for DPeCF3. This increase in ICT reaction efficiency is accompanied by a small but significant decrease of $380 \mathrm{~cm}^{-1}$ for $\Delta E\left(S_{1}, S_{2}\right)$ when going from DMCF3 to DPeCF3. Because of their relatively large $\Delta E\left(S_{1}, S_{2}\right)$ gap, the molecules DXCF3 are
\end{abstract}

\footnotetext{
${ }^{*}$ Permanent affiliation: B.I. Stepanov Institute of Physics, National Academy of Sciences of Belarus, 220072 Minsk, Belarus.

${ }^{\dagger}$ corresponding author; e-mail: kzachar@gwdg.de
} 
suitable for an investigation of the excitation wavelength dependence of the intramolecular charge transfer reaction. For DPrCF3 in MeCN, the same $\Phi^{\prime}(\mathrm{ICT}) / \Phi(\mathrm{LE})$ is found for excitation in the $S_{1}$ or in the $S_{2}$ manifold. A similar result is obtained with DMABN in tetrahydrofuran. From these experiments it is concluded that for DPrCF3 and DMABN the intramolecular charge transfer reaction follows an adiabatic pathway. After excitation to a Franck-Condon $S_{n}(\mathrm{FC})$ state, the electron donor/acceptor molecules first undergo ultrafast internal conversion to the relaxed $\operatorname{LE}\left(S_{1}\right)$ state, from which precursor the reaction proceeds to the ICT state. Experimental evidence for a nonadiabatic ICT reaction pathway, going directly from $S_{2}(\mathrm{FC})$ via an $S_{2} / S_{1}$ conical intersection to the LE and ICT state, is not found. This pathway would lead to an enhancement of the ICT population, i.e., to an increase in $\Phi^{\prime}(\mathrm{ICT}) / \Phi(\mathrm{LE})$, as compared with the adiabatic LE $\rightarrow$ ICT reaction.

PACS numbers: 33.20.-t, 33.50.- $\mathrm{j}$

\section{Introduction}

In the discussions around the intramolecular charge transfer (ICT) in the excited singlet state with electron donor(D)/acceptor(A) molecules such as 4-(dimethylamino)benzonitrile (DMABN), two issues are at present in the center of the investigations.

The major issue is perhaps still the molecular structure of the ICT state. For this state, two models making a statement on the twist angle of the amino group with respect to the phenyl ring have survived the debate since the discovery $[1,2]$ of the dual fluorescence of DMABN and its derivatives in 1959: the TICT (twisted ICT) and PICT (planar ICT) models [3-5]. In the TICT structure, a perpendicular, i.e., fully twisted $\left(90^{\circ}\right)[3,6]$, amino group is assumed [7], whereas in the PICT model $[4,5,8,9]$ such a $90^{\circ}$ twist is considered not to be necessary for an efficient ICT reaction of the D/A molecules. Rather than emphasizing the magnitude of the twist angle, the PICT model assumes a nonzero electronic coupling between the amino (D) and benzonitrile (A) moieties in the ICT state, which coupling is absent (or minimal) in the classical TICT structure. An essential requirement for the occurrence of ICT from the PICT point of view is the presence of a sufficiently small energy gap $\Delta E\left(S_{1}, S_{2}\right)$ between the two lowest excited singlet states. A specific molecular structure of the ICT state, such as a quinoidal classical resonance structure for the ICT state of DMABN [8], is not part of the PICT model.

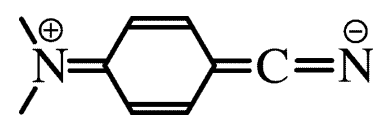

A second issue is related to the detailed reaction pathway of the ICT reaction of D/A molecules such as DMABN after preparing the Franck-Condon $S_{n}(\mathrm{FC})$ state upon light excitation. According to the present authors and previous publications, internal conversion (IC) follows excitation and the ICT reaction then proceeds from the relaxed locally excited (LE) state as the precursor, in an adiabatic 
reaction pathway $[4,5,8-10]$. An alternative mechanism was proposed by Fuß and co-workers on the basis of their femtosecond nonresonant multiphoton ionization experiments with DMABN and 1-methyl-6-cyano-1,2,3,4-tetrahydroquinoline (NMC6) in the gas phase [11, 12]. In their view, the molecule excited to the $S_{2}(\mathrm{FC})$ state does not undergo IC, but passes through a conical intersection (CI) of the $S_{2}$ and $S_{1}$ potential energy surfaces and from there can form the ICT as well as the LE state. In this nonadiabatic reaction pathway, LE is clearly not the ICT precursor and the ICT state can even be populated in principle under endothermic ICT conditions, i.e., in cases where the energy of the ICT state is higher than that of LE. The experimental finding that ICT emission is not observed with DMABN in the gas phase or in alkane solvents nor with NMC6 in any medium was explained by assuming a very efficient subpicosecond ICT $\rightarrow$ LE reaction, thereby reducing the emission quantum yield $\Phi^{\prime}(\mathrm{ICT})$ of the primarily populated ICT state to effectively zero, precluding experimental verification of its existence. Obviously, a reaction via the $S_{2} / S_{1}$ CI would not be possible for excitation energies smaller than the energy of the $S_{2}(\mathrm{FC})$ state. This condition will be investigated in the present paper by studying the excitation wavelength dependence of the ICT and LE dual fluorescence spectra of DMABN in tetrahydrofuran (THF) and of N,N-di- $n$-propyl-4-(trifluoromethyl)aniline (DPrCF3) in acetonitrile $(\mathrm{MeCN})$.

The Fuß mechanism $[11,12]$ has been adopted in the discussion of the results of recent calculations with DMABN, NMC6 and 1-tert-butyl-6-cyano-1,2,3,4-tetrahydroquinoline (NTC6) [13-16]. It was assumed that the $S_{2}$ state is predominantly prepared during the excitation process, due to the substantially larger transition dipole moment of $S_{0} \rightarrow S_{2}$ as compared with $S_{0} \rightarrow S_{1}$ [17-19]. The possibility to excite the $\mathrm{D} / \mathrm{A}$ molecule exclusively into $S_{1}$, precluding access to the $S_{2} / S_{1}$ CI, was not explicitly taken into account.

\section{Experimental section}

\subsection{Molecules and solvents}

DMABN was made from 4-bromo-N,N-dimethylaniline (Aldrich) in a reaction with $\mathrm{CuCN}$ [20]. 4-(Methylamino)benzonitrile (MABN) was made from 4-aminobenzonitrile $(\mathrm{ABN})$ and trifluoroacetic anhydride giving 4-(trifluoroacetamido)benzonitrile, which was converted into MABN with iodomethane [21]. ABN was made from 4-bromoaniline and $\mathrm{CuCN}$ in N-methyl-2-pyrrolidone. The N,N-di-n-alkyl-4-(trifluoromethyl)anilines (DXCF3) and the $\mathrm{N}-n$-alkyl-4-(trifluoromethyl)anilines (XCF3), with X = methyl (M), ethyl (E), $n$-propyl (Pr) and $n$-pentyl (Pe), were synthesized from 4-(trifluoromethyl)aniline (Aldrich) and the corresponding $n$-alkyl iodide (Fluka) in absolute methanol. For these molecules, high pressure liquid chromatography (HPLC) was the last purification step. The solvents acetonitrile (Merck, Uvasol), ethyl cyanide (EtCN, Merck, for synthesis), diethyl ether (DEE, Merck, Uvasol), and tetrahydrofu- 
ran (THF, Merck, Uvasol) were chromatographed over $\mathrm{Al}_{2} \mathrm{O}_{3}$, whereas $n$-hexane (Merck, Uvasol) was used as received. The solutions, with an optical density between 0.4 and 0.6 at the maximum of the first band in the absorption spectrum, were deaerated by bubbling with nitrogen for 15 minutes.

\subsection{Absorption and fluorescence spectra}

The absorption spectra were run on a Cary 500 spectrometer. The fluorescence spectra were measured with a quantum-corrected (modified [10]) Fluoromax 3 spectrofluorometer. The fluorescence quantum yields $\Phi_{\mathrm{f}}$, with an estimated reproducibility of 2 percent, were determined with quinine sulfate in $1.0 \mathrm{~N} \mathrm{H}_{2} \mathrm{SO}_{4}$ as a standard $\left(\Phi_{\mathrm{f}}=0.546\right.$ at $\left.25^{\circ} \mathrm{C}\right)[22]$, with equal optical density at the excitation wavelength. The difference in refractive index between the standard solution and the solvents was not taken into account.

\section{Results and discussion}

3.1. Absorption and fluorescence spectra of DMABN and DMCF3 in n-hexane, diethyl ether and acetonitrile

\subsection{1. $D M A B N$}

The absorption spectra of DMABN in three solvents of different polarity, $n$-hexane $(\varepsilon=1.88)$, DEE $(\varepsilon=4.23)$ and $\mathrm{MeCN}(\varepsilon=36.7)$ at $25^{\circ} \mathrm{C}$, are shown in Fig. 1. For DMABN in $n$-hexane, the weak and structured $S_{1}$ band is clearly visible at the low-energy side of the main, more strongly allowed, $S_{2}$ absorption $[17,18]$.

TABLE I

Absorption maximum $\tilde{\nu}^{\max }(\mathrm{abs})$, emission maxima $\tilde{\nu}^{\max }(\mathrm{LE})$ and $\tilde{\nu}^{\max }(\mathrm{ICT})$, energy $E\left(S_{1}\right)$, energy gap $\Delta E\left(S_{1}, S_{2}\right)$ and ICT/LE fluorescence quantum yield ratio $\Phi^{\prime}(\mathrm{ICT}) / \Phi(\mathrm{LE})$ of DMABN and DMCF3 in $n$-hexane, DEE and MeCN at $25^{\circ} \mathrm{C}$, see Figs. 1 and 2.

\begin{tabular}{|c|c|c|c|c|c|c|}
\hline & \multicolumn{3}{|c|}{ DMABN } & \multicolumn{3}{|c|}{ DMCF3 } \\
\hline & $n$-hexane ${ }^{a}$ & DEE & $\mathrm{MeCN}$ & $n$-hexane & DEE & $\mathrm{MeCN}$ \\
\hline$\tilde{\nu}^{\max }\left(S_{2}, \mathrm{abs}\right)\left[\mathrm{cm}^{-1}\right]$ & 35530 & 35020 & 34220 & 38020 & 37710 & 37280 \\
\hline$\tilde{\nu}\left(S_{1}^{0}, \mathrm{abs}\right)\left[\mathrm{cm}^{-1}\right]^{b}$ & 31640 & 31360 & 31090 & 32150 & 31890 & 31530 \\
\hline$\tilde{\nu}^{\max }(\mathrm{LE})\left[\mathrm{cm}^{-1}\right]$ & 29570 & 28700 & 27720 & 30080 & 29380 & 28240 \\
\hline$\tilde{\nu}^{\max }(\mathrm{ICT})\left[\mathrm{cm}^{-1}\right]$ & - & 23840 & 20330 & - & - & 22120 \\
\hline$E\left(S_{1}\right)\left[\mathrm{cm}^{-1}\right]^{c}$ & 31840 & 31360 & 29980 & 32340 & 31850 & 31170 \\
\hline$\Delta E\left(S_{1}, S_{2}\right)\left[\mathrm{cm}^{-1}\right]^{d}$ & 3890 & 3660 & 3130 & 5870 & 5820 & 5750 \\
\hline$\Phi^{\prime}(\mathrm{ICT}) / \Phi(\mathrm{LE})$ & 0 & 0.30 & 39.5 & 0 & 0 & 0.06 \\
\hline $\begin{array}{l}{ }^{a} \text { For DMABN in po } \\
{ }^{b} \text { Energy of the first } \\
\text { between the absorpt } \\
\tilde{\nu}^{\max }\left(S_{2}, \text { abs }\right) \text { and } \nu(\end{array}$ & hylene ( $\mathrm{R}$ & [25]). & ctra. & (1) & $00 \mathrm{~cm}$ & $\begin{array}{l}\left(S_{2}\right) \text {. } \\
\text { ssing } \\
\text { ween }\end{array}$ \\
\hline
\end{tabular}




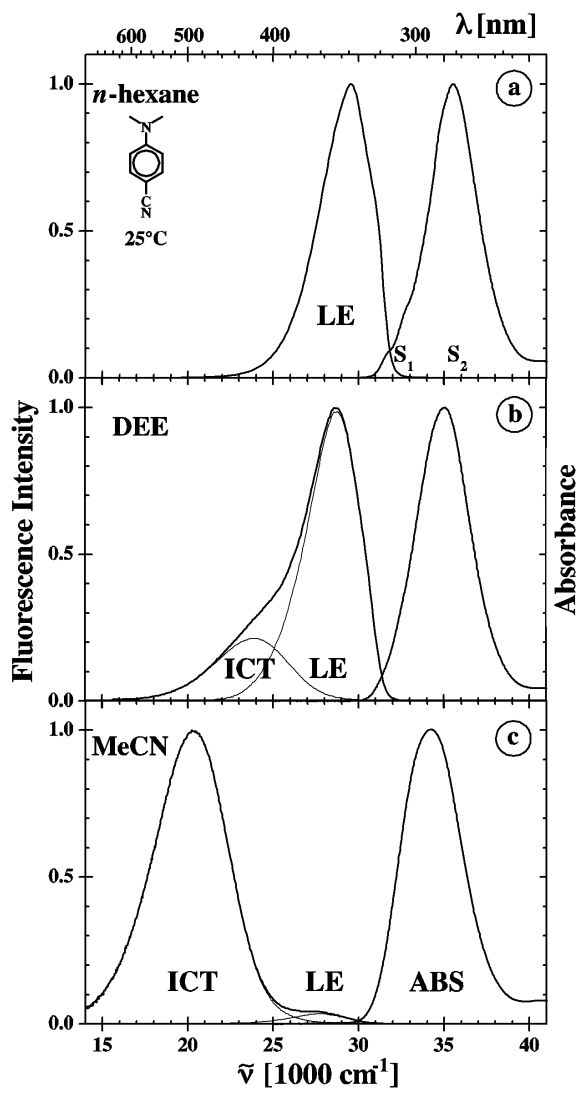

Fig. 1. Fluorescence (LE and ICT) and absorption (ABS) spectra of DMABN in (a) $n$-hexane, (b) DEE and (c) MeCN at $25^{\circ} \mathrm{C}$. The $S_{1}$ and $S_{2}$ absorption bands (see text) are indicated in (a). The spectral separation of the LE and ICT bands of the overall fluorescence spectrum has been carried out by taking the fluorescence spectrum of MABN as that of the LE emission of DMABN.

Whereas the $S_{0} \rightarrow S_{1}$ transition corresponds to the weak $S_{0} \rightarrow{ }^{1} L_{\mathrm{b}}$ absorption of benzene, the $S_{2}$ state has an important charge transfer (CT) admixture to the ${ }^{1} L_{\mathrm{a}}$ state of benzene origin [19].

Due to the larger dipole moment of the $S_{2}$ state [17-19], the $S_{1}$ band more and more disappears with increasing solvent polarity under the $S_{2}$ absorption, as seen for DEE (Fig. 1b) and MeCN (Fig. 1c). In the last solvent of high polarity, there is no longer a sign of the $S_{1}$ band in the absorption spectrum of DMABN. This means that the energy gap $\Delta E\left(S_{1}, S_{2}\right)$ of DMABN decreases when the solvent polarity becomes larger (Table I) $[17,18]$. As emphasized in the PICT model $[4,5,8,9]$, this decrease in $\Delta E\left(S_{1}, S_{2}\right)$ leads to the appearance of an ICT fluorescence band to the red of the LE emission. Whereas there is no indication of the presence of ICT fluorescence for DMABN in $n$-hexane (Fig. 1a) [3, 9, 10, 23] 
ICT emission is clearly present in DEE and has become the major fluorescence band in MeCN [3, 9, 10, 24, 25], with ICT/LE fluorescence quantum yield ratios $\Phi^{\prime}(\mathrm{ICT}) / \Phi(\mathrm{LE})$ of 0.30 in DEE and 39.5 in MeCN (Table I).

\subsection{2. $D M C F 3$}

In the absorption spectra of $\mathrm{N}, \mathrm{N}-\mathrm{di}-n$-methyl-4-(trifluoromethyl)aniline (DMCF3) in $n$-hexane, DEE and $\mathrm{MeCN}$ at $25^{\circ} \mathrm{C}$ (Fig. 2), the weak and struc-

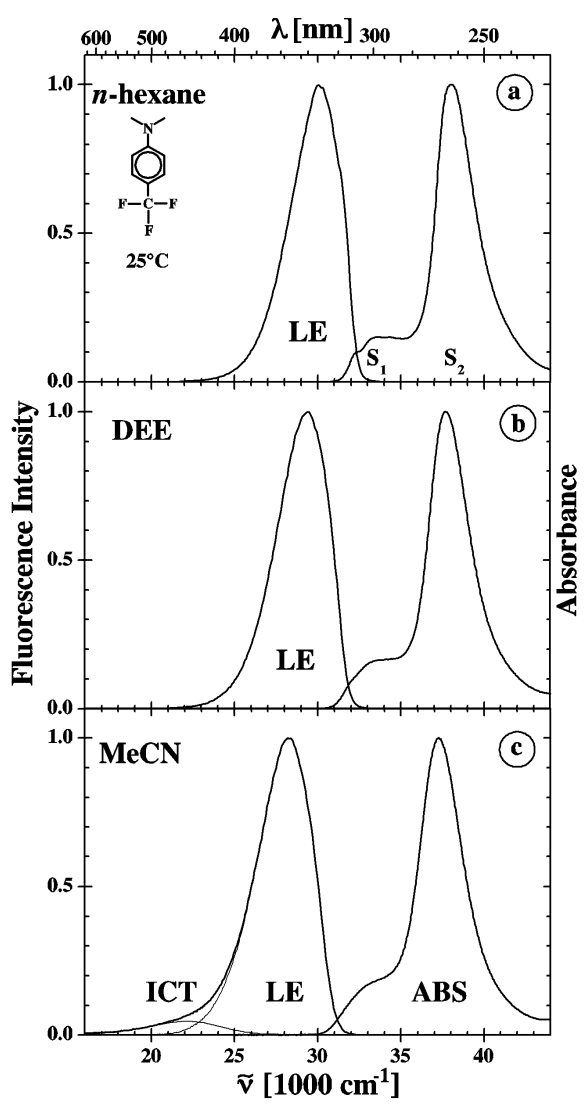

Fig. 2. Fluorescence (LE and ICT) and absorption (ABS) spectra of DMCF3 in (a) $n$-hexane, (b) DEE and (c) $\mathrm{MeCN}$ at $25^{\circ} \mathrm{C}$. The $S_{1}$ and $S_{2}$ absorption bands (see text) are indicated in (a). The separation of the overall fluorescence spectrum into the LE and ICT contributions has been carried out by taking the fluorescence spectrum of N,N-n-methyl-4-(trifluoromethyl)aniline (MCF3) as that of the LE emission of DMCF3.

tured $S_{1}$ absorption is clearly separated from the main $S_{2}$ absorption, with a $\Delta E\left(S_{1}, S_{2}\right)$ gap of $5870 \mathrm{~cm}^{-1}$ in $n$-hexane, considerably larger than the gap of $3890 \mathrm{~cm}^{-1}$ for DMABN in this solvent (Table I). The relatively large $\Delta E\left(S_{1}, S_{2}\right)$ has as its consequence, that the ICT emission for DMCF3 $\left(\Phi^{\prime}(\mathrm{ICT}) / \Phi(\mathrm{LE})=\right.$ $0.06)$ is very weak even in the strongly polar MeCN and is absent in $n$-hexane and 
DEE, clearly different from the observations with DMABN (Fig. 1 and Table I). The results with DMABN and DMCF3 show that $\Delta E\left(S_{1}, S_{2}\right)$ is the determining factor for the occurrence of ICT and dual fluorescence with D/A molecules, in accordance with the PICT approach [4, 5, 8, 9].

The relatively large $\Delta E\left(S_{1}, S_{2}\right)$ gap of the DXCF3s opens up the possibility to investigate the dependence of the ICT reaction efficiency $\left(\Phi^{\prime}(\mathrm{ICT}) / \Phi(\mathrm{LE})\right)$ on excitation wavelength $\lambda$ (exc), by varying $\lambda$ (exc) over the $S_{1}$ and $S_{2}$ bands in the absorption spectra. This dependence will be investigated here and discussed in connection with adiabatic and nonadiabatic ICT reaction pathways.

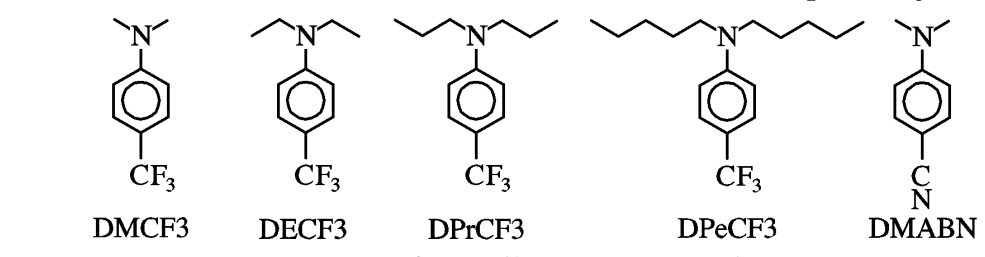

Molecular structures of DXCF3 and DMABN

3.2. Different reduction potentials for benzonitrile and (trifluoromethyl)benzene. $D X C F 3$ versus $D X A B N$

At least part of the difference between the ICT efficiency and energetics (including $\Delta E\left(S_{1}, S_{2}\right)$ ) of DXCF3 and the corresponding 4-(dialkylamino)benzonitriles (DXABN) comes from the more negative reduction potential $E\left(\mathrm{~A}^{-} / \mathrm{A}\right)$ (smaller electron affinity) of the (trifluoromethyl)benzene as compared with the benzonitrile electron acceptor moieties: $2.54 \mathrm{~V}$ against $2.30 \mathrm{~V}$ vs. saturated calomel electrode (SCE) [26]. For a series of DXABN D/A molecules, a correlation between the energy of the ICT state $E(\mathrm{ICT})$ and the difference between the oxidation and reduction potentials of the D and A subgroups has been established, with a correlation coefficient $f=0.29$, see Eq. 1 [27]:

$$
E(\mathrm{ICT})=f\left[E\left(\mathrm{D}^{+} / \mathrm{D}\right)-E\left(\mathrm{~A}^{-} / \mathrm{A}\right)\right]+\mathrm{C} .
$$

\subsection{Absorption and fluorescence spectra of DMCF3, DECF3, DPrCF3, and DPeCF3 in n-hexane}

The absorption and fluorescence spectra of the N,N-di- $n$-alkyl-4-(trifluoromethyl)anilines DXCF3 with $\mathrm{X}=\mathrm{M}$ (methyl), $\mathrm{E}$ (ethyl), Pr ( $n$-propyl) and $\mathrm{Pe}(n$-pentyl $)$ in $n$-hexane at $25^{\circ} \mathrm{C}$ are shown in Fig. 3 . As already discussed for DMCF3 (Fig. 2a), the absorption spectra of all DXCF3 consist of a main structureless $S_{2}$ absorption band and a weak structured $S_{1}$ band at lower energies (Table II). The energy difference between the first absorption peak of $S_{1}\left(\tilde{\nu}\left(S_{1}^{0}\right.\right.$,abs $\left.)\right)$ and the maximum of the $S_{2}$ absorption band $\tilde{\nu}^{\max }\left(S_{2}\right.$,abs), taken as an approximation for $\Delta E\left(S_{1}, S_{2}\right)$, becomes somewhat smaller with increasing amino-alkyl chain length, from $5870 \mathrm{~cm}^{-1}$ for DMCF3 to $5490 \mathrm{~cm}^{-1}$ for DPeCF3 (Table II). For all four DXCF3 in $n$-hexane at $25^{\circ} \mathrm{C}$, the fluorescence spectrum consists of a single $\mathrm{LE}$ emission, without any indication of the presence of ICT fluorescence. 


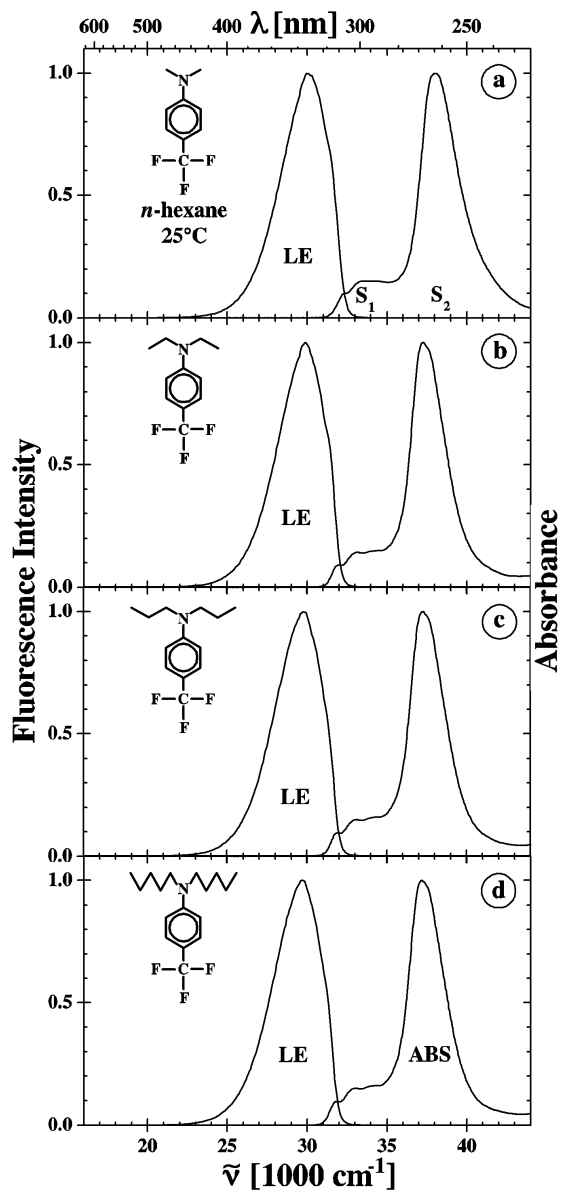

Fig. 3. Fluorescence (LE and ICT) and absorption (ABS) spectra of (a) DMCF3, (b) DECF3, (c) DPrCF3 and (d) DPeCF3 in $n$-hexane at $25^{\circ} \mathrm{C}$. The $S_{1}$ and $S_{2}$ absorption bands (see text) are indicated in (a).

\subsection{Absorption and fluorescence spectra of DMCF3, DECF3, DPrCF3, and DPeCF3 in acetonitrile}

The absorption spectra of the DXCF3s in MeCN at $25^{\circ} \mathrm{C}$ in Fig. 4 are similar to those in $n$-hexane (Fig. 3), with somewhat smaller energies for $\tilde{\nu}^{\max }\left(S_{2}\right.$, abs) and $\tilde{\nu}^{\max }(\mathrm{LE})$, see Table II, due to the larger solvent polarity. Different from the results in $n$-hexane, the fluorescence spectra of the DXCF3s in MeCN show dual fluorescence. The ratio $\Phi^{\prime}(\mathrm{ICT}) / \Phi(\mathrm{LE})$ increases when the amino-alkyl chain becomes longer, in particular from DMCF3 to DECF3: 0.06 (DMCF3), 0.50 (DECF3), 0.54 (DPrCF3), 0.64 (DPeCF3) (Table II). A similar increase in $\Phi^{\prime}(\mathrm{ICT}) / \Phi(\mathrm{LE})$ with increasing alkyl chain length has been found with DXABN and derivatives in toluene [27]. 
TABLE II

Absorption maximum $\tilde{\nu}^{\max }(\mathrm{abs})$, extinction coefficient $\varepsilon^{\max }$, emission maxima $\tilde{\nu}^{\max }(\mathrm{LE})$ and $\tilde{\nu}^{\max }(\mathrm{ICT})$, energy $E\left(S_{1}\right)$, energy gap $\Delta E\left(S_{1}, S_{2}\right)$ and ICT/LE fluorescence quantum yield ratio $\Phi^{\prime}(\mathrm{ICT}) / \Phi(\mathrm{LE})$ of the DXCF3 with $\mathrm{X}=\mathrm{M}$ (methyl), $\mathrm{E}$ (ethyl), Pr (n-propyl) and Pe ( $n$-pentyl) in $n$-hexane and MeCN at $25^{\circ} \mathrm{C}$, see Figs. 3 and 4 .

\begin{tabular}{|c|c|c|c|c|}
\hline & \multicolumn{2}{|c|}{ DMCF3 } & \multicolumn{2}{|c|}{ DECF3 } \\
\hline & $n$-hexane & $\mathrm{MeCN}$ & $n$-hexane & $\mathrm{MeCN}$ \\
\hline$\tilde{\nu}^{\max }\left(S_{2}, \mathrm{abs}\right)\left[\mathrm{cm}^{-1}\right]$ & 38020 & 37280 & 37250 & 36680 \\
\hline$\tilde{\nu}\left(S_{1}^{0}, \mathrm{abs}\right)\left[\mathrm{cm}^{-1}\right]^{a}$ & 32150 & $(31530)$ & 31840 & 31190 \\
\hline$\varepsilon^{\max }\left[\mathrm{M}^{-1} \mathrm{~cm}^{-1}\right]$ & 24200 & 20860 & & \\
\hline$\tilde{\nu}^{\max }(\mathrm{LE})\left[\mathrm{cm}^{-1}\right]$ & 30080 & 28240 & 29890 & 28300 \\
\hline$\tilde{\nu}^{\max }(\mathrm{ICT})\left[\mathrm{cm}^{-1}\right]$ & - & 22120 & - & 22630 \\
\hline$E\left(S_{1}\right)\left[\mathrm{cm}^{-1}\right]^{b}$ & 32340 & 31170 & 32080 & 31070 \\
\hline$\Delta E\left(S_{1}, S_{2}\right)\left[\mathrm{cm}^{-1}\right]^{c}$ & 5870 & 5750 & 5410 & 5490 \\
\hline \multirow[t]{3}{*}{$\Phi^{\prime}(\mathrm{ICT}) / \Phi(\mathrm{LE})$} & 0 & 0.06 & 0 & 0.50 \\
\hline & \multicolumn{2}{|c|}{ DPrCF3 } & \multicolumn{2}{|c|}{ DPeCF3 } \\
\hline & $n$-hexane & $\mathrm{MeCN}$ & $n$-hexane & $\mathrm{MeCN}$ \\
\hline$\tilde{\nu}^{\max }\left(S_{2}, \mathrm{abs}\right)\left[\mathrm{cm}^{-1}\right]$ & 37240 & 36700 & 37200 & 36650 \\
\hline$\tilde{\nu}\left(S_{1}^{0}, \mathrm{abs}\right)\left[\mathrm{cm}^{-1}\right]^{a}$ & 31730 & 31320 & 31710 & 31280 \\
\hline$\varepsilon^{\max }\left[\mathrm{M}^{-1} \mathrm{~cm}^{-1}\right]$ & & 22690 & & \\
\hline$\tilde{\nu}^{\max }(\mathrm{LE})\left[\mathrm{cm}^{-1}\right]$ & 29800 & 28020 & 29700 & 28150 \\
\hline$\tilde{\nu}^{\max }(\mathrm{ICT})\left[\mathrm{cm}^{-1}\right]$ & - & 23160 & & 23300 \\
\hline$E\left(S_{1}\right)\left[\mathrm{cm}^{-1}\right]^{b}$ & 31990 & 31010 & 31910 & 31020 \\
\hline$\Delta E\left(S_{1}, S_{2}\right)\left[\mathrm{cm}^{-1}\right]^{c}$ & 5510 & 5380 & 5490 & 5370 \\
\hline$\Phi^{\prime}(\mathrm{ICT}) / \Phi(\mathrm{LE})$ & 0 & 0.54 & 0 & 0.64 \\
\hline
\end{tabular}

${ }^{a}$ Energy of the first vibrational peak of the $S_{1}$ absorption band. ${ }^{b}$ Crossing between the absorption and fluorescence spectra. ${ }^{c}$ Energy difference between $\tilde{\nu}^{\max }\left(S_{2}\right.$,abs $)$ and $\tilde{\nu}\left(S_{1}^{0}\right.$, abs $)$.

3.5. $\Phi^{\prime}(I C T) / \Phi(L E)$ of $D P r C F 3$ in ethyl cyanide as a function of temperature. The Stevens-Ban plot

The temperature dependence of the ratio $\Phi^{\prime}(\mathrm{ICT}) / \Phi(\mathrm{LE})$ of DPrCF3 in ethyl cyanide (EtCN) over a large temperature range between the boiling and melting points of the solvent is plotted in Fig. 5.

The expression for $\Phi^{\prime}(\mathrm{ICT}) / \Phi(\mathrm{LE})$ is given by Eq. (2), for the ICT reaction mechanism represented by Scheme 1 .

$$
\frac{\Phi^{\prime}(\mathrm{ICT})}{\Phi(\mathrm{LE})}=\frac{k_{\mathrm{f}}^{\prime}(\mathrm{ICT})}{k_{\mathrm{f}}(\mathrm{LE})} \frac{k_{\mathrm{a}}}{k_{\mathrm{d}}+1 / \tau_{0}^{\prime}(\mathrm{ICT})} .
$$




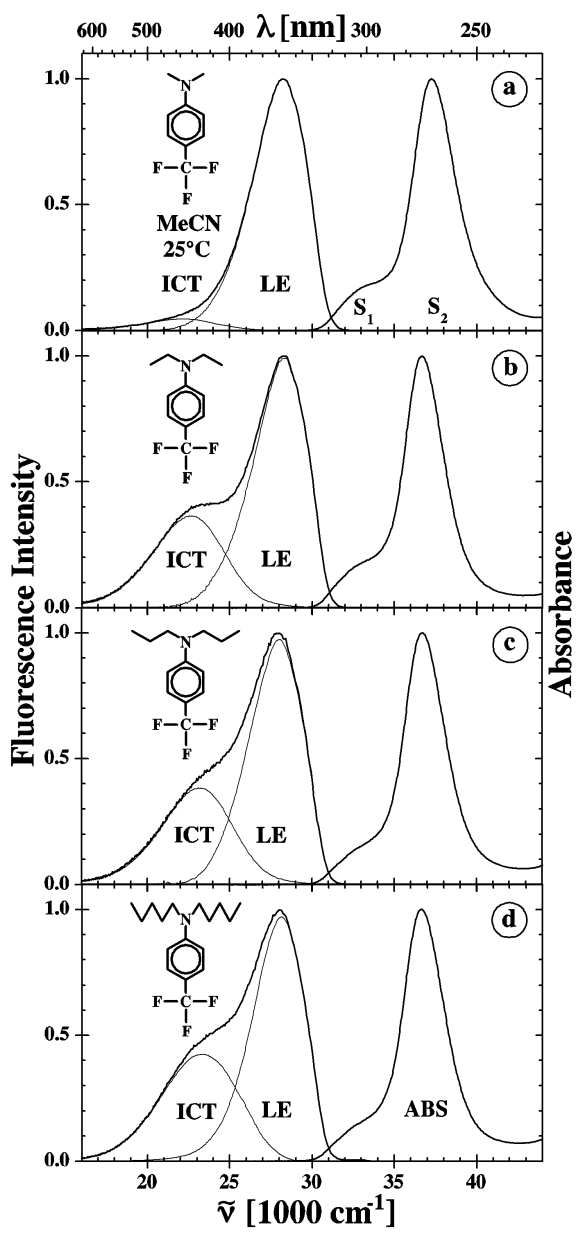

Fig. 4. Fluorescence (LE and ICT) and absorption (ABS) spectra of (a) DMCF3, (b) DECF3, (c) DPrCF3 and (d) DPeCF3 in MeCN at $25^{\circ} \mathrm{C}$. The $S_{1}$ and $S_{2}$ absorption bands (see text) are indicated in (a). The separation of the overall fluorescence spectrum into the LE and ICT contributions has been carried out by taking the fluorescence spectrum of the corresponding N,N-n-alkyl-4- (trifluoromethyl)anilines (XCF3) in MeCN as that of the LE emission of DXCF3.

Scheme 1

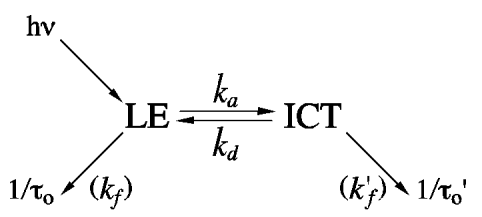

In Eq. (2) and Scheme 1, $k_{\mathrm{a}}$ and $k_{\mathrm{d}}$ are the rate constants of the forward and backward ICT reaction, $\tau_{0}(\mathrm{LE})$ and $\tau_{0}^{\prime}(\mathrm{ICT})$ are the fluorescence lifetimes, whereas $k_{\mathrm{f}}(\mathrm{LE})$ and $k_{\mathrm{f}}^{\prime}(\mathrm{ICT})$ are the radiative rate constants. 


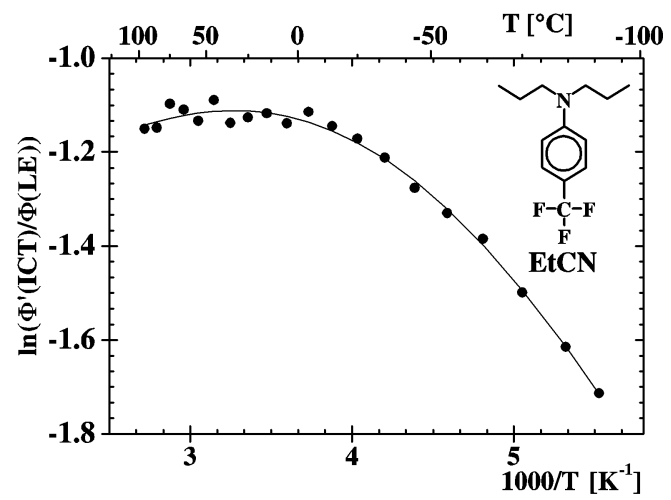

Fig. 5. Stevens-Ban plot of the ICT/LE fluorescence quantum yield ratio $\Phi^{\prime}(\mathrm{ICT}) / \Phi(\mathrm{LE})$ of DPrCF3 in EtCN. From the HTL slope, the ICT reaction enthalpy $\Delta H$ is obtained, whereas the LTL slope yields the activation energy $E_{\text {a }}$ of the LE $\rightarrow$ ICT reaction, see text (Eq. (2)). The line through the data points represents the fitting by employing Eq. (2), giving the following data: $\Delta H=-2.7 \mathrm{~kJ} / \mathrm{mol}$ and $E_{\mathrm{a}}=5.7 \mathrm{~kJ} / \mathrm{mol}$.

A complete so-called Stevens-Ban [28] plot of $\Phi^{\prime}(\mathrm{ICT}) / \Phi(\mathrm{LE})$ versus the reciprocal absolute temperature involves a high-temperature limit (HTL), where $k_{\mathrm{d}} \gg 1 / \tau_{0}^{\prime}(\mathrm{ICT})$, and a low-temperature limit (LTL), where $k_{\mathrm{d}} \ll 1 / \tau_{0}^{\prime}(\mathrm{ICT})$ [10, 28-30]. From the Stevens-Ban plot, the activation energies of the forward $\left(E_{\mathrm{a}}\right)$ and backward $\left(E_{\mathrm{d}}\right)$ ICT reaction (Scheme 1$)$, as well as the ICT reaction enthalpy $\Delta H\left(=E_{\mathrm{a}}-E_{\mathrm{d}}\right)$ can be obtained. Under HTL conditions, the slope of the Stevens-Ban plot is equal to $-\Delta H / R$, whereas the slope is $E_{\mathrm{a}} / R$ for the LTL [10, 28-30].

It appears from Fig. 5, that $\Phi^{\prime}(\mathrm{ICT}) / \Phi(\mathrm{LE})$ only slightly decreases over the temperature range from 0 to $95^{\circ}$, which indicates that the $\Delta H$ for DPrCF3 in EtCN is small. By fitting all the data points plotted in Fig. 5 by Eq. (2) the following values are determined: $\Delta H=-2.7 \mathrm{~kJ} / \mathrm{mol}$ and $E_{\mathrm{a}}=5.7 \mathrm{~kJ} / \mathrm{mol}$. It is thereby assumed that $k_{\mathrm{f}}^{\prime}(\mathrm{ICT}) / k_{\mathrm{f}}(\mathrm{LE})$ and also $\tau_{0}^{\prime}(\mathrm{ICT})(\mathrm{Eq} .(2))$ do not depend on temperature [10].

\subsection{Adiabatic reaction pathway for intramolecular charge transfer in the excited singlet state}

Generally, after light absorption into a higher excited singlet state $S_{n}(n>1)$, molecules undergo ultrafast IC to the lowest excited singlet state $S_{1}$ : the Kasha rule [31]. This means that excited state processes, such as the ICT reaction of DMABN, originate from the relaxed $S_{1}(\mathrm{LE})$ state precursor (Fig. 6), as IC is much faster than the ICT reaction, which process has for DMABN in MeCN at $25^{\circ} \mathrm{C}$ a reaction time of $4 \mathrm{ps}[10]$. In support of this reaction pathway, the time-resolved stimulated emission spectrum of DMABN in MeCN at time zero is that of the LE state, indicating that LE is the precursor of the ICT state and that a direct pathway from $S_{2}$ to ICT, bypassing LE, does not take place [10]. A similar 


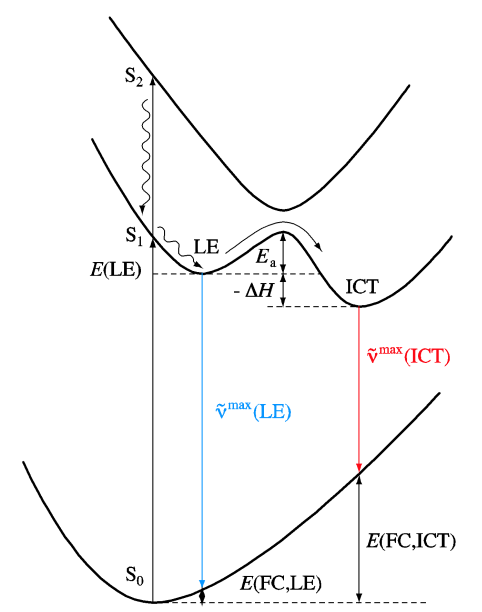

Fig. 6. Adiabatic ICT reaction pathway. Potential energy surfaces for the ground state $S_{0}$ and the excited singlet states $S_{1}$ and $S_{2}$. When excited with energies equal to or larger than $E\left(S_{2}\right)$ into the manifold of the $S_{2}$ state, the system subsequently relaxes by internal conversion to the equilibrated LE state, with an energy $E(\mathrm{LE})$ above $S_{0}$. The ICT reaction proceeds from the LE to the ICT state, with a reaction barrier $E_{\mathrm{a}}$ and an enthalpy difference $\Delta H$. Fluorescence from the LE and ICT states, with emission maxima $\tilde{\nu}^{\max }(\mathrm{LE})$ and $\tilde{\nu}^{\max }(\mathrm{ICT})$, reaches the corresponding Franck-Condon states $E(\mathrm{FC}, \mathrm{LE})$ and $E(\mathrm{FC}, \mathrm{ICT})$.

observation and conclusion was made for the time-resolved fluorescence spectrum of NTC6 in $n$-hexane [32]. In support of this conclusion, there is also no sign of an important ICT contribution in the excited state absorption spectra of DMABN in $n$-hexane or MeCN at 200 fs after excitation [10, 32].

\subsection{Nonadiabatic ICT reaction. Formation of ICT state via the $S_{2} / S_{1}$ conical intersection, bypassing $S_{1}(L E)$}

Based on femtosecond nonresonant multiphoton ionization experiments with DMABN and NMC6 in the gas phase, as mentioned in the Introduction, Fuß and co-workers came to the conclusion that the ICT reaction of these molecules does not follow an adiabatic pathway $[11,12]$. In their interpretation, after excitation to the $S_{2}$ or higher $S_{n}$ state, the molecules pass through an $S_{2} / S_{1}$ CI and from there are distributed over the ICT and LE states (Fig. 7), even when the ICT state would have a higher energy than LE. The absence of ICT emission in the fluorescence spectra of NMC6 (in the vapor phase and all solvents) or of DMABN in the gas phase and $n$-hexane $[10,17,23,32,33]$, is then explained by postulating a very efficient ICT $\rightarrow$ LE back reaction over a low barrier on a subpicosecond timescale, with which the intrinsically slow ICT fluorescence (radiative rate $\approx 10^{7} \mathrm{~s}^{-1}$ ) [10] cannot compete. As mentioned in the previous section, time-resolved fluorescence spectra of DMABN in $n$-hexane and MeCN and of NTC6 in $n$-hexane with a time resolution of $3 \mathrm{ps}$, however, do not reveal an ICT emission at time zero. 


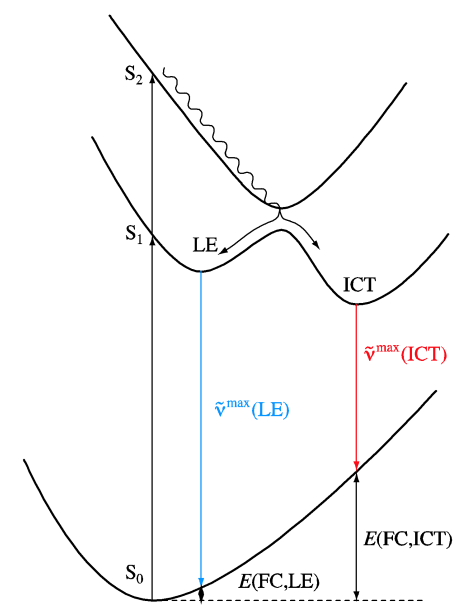

Fig. 7. Nonadiabatic ICT reaction pathway. Potential energy surfaces for the ground state $S_{0}$ and the excited states $S_{1}$ and $S_{2}$. When excited into the $S_{2}$ manifold, the system passes through an $S_{2} / S_{1}$ conical intersection, from which the LE as well as the ICT state is directly populated. In this reaction pathway, the relaxed LE state is not the ICT precursor, as is the case for the adiabatic reaction pathway in Fig. 6. See the caption of Fig. 6.

This nonadiabatic reaction mechanism has been discussed in connection with recent calculations on the $\mathrm{LE} \rightarrow \mathrm{ICT}$ reaction of DMABN and NTC6, to explain how an ICT state can be populated even in molecules for which the $S_{1}(\mathrm{LE}) \rightarrow$ ICT reaction is endothermic [13-16]. In the nonadiabatic process, favored in the interpretation of these calculations, the barrier height between ICT and LE is an important issue, as a low ICT $\rightarrow$ LE barrier was taken as the explanation for the absence of ICT emission in the experimental fluorescence spectra [10, 23, 32, 33] of DMABN and NMC6 in alkane solvents as well as in the vapor phase. From the computed schematic potential energy profiles for DMABN (i.e., in the gas phase at $0 \mathrm{~K}$ ) [14], the ICT $\rightarrow$ LE reaction barrier amounts to $65.7 \mathrm{~kJ} / \mathrm{mol}$, which barrier would completely prevent the disappearance of ICT emission in the fluorescence spectrum by subpicosecond thermal depopulation. It was stated in Ref. [14], however, that the ICT reaction with DMABN in solution takes place via an adiabatic pathway, with LE as the precursor, leaving open the possibility that the nonadiabatic CI-mechanism with direct formation of the ICT state only takes place in the gas phase.

\subsection{Excitation wavelength dependence of the ICT reaction}

When it is assumed that the ICT reaction with D/A molecules such as DMABN and NTC6 follows a nonadiabatic reaction pathway from the $S_{2}(\mathrm{FC})$ state reached on light absorption, via the $S_{2} / S_{1}$ CI to the LE and ICT states (Fig. 7), such a process is clearly not possible for excitation energies lower 
than $E\left(S_{2}\right)$. We therefore carried out fluorescence experiments with DMABN and DPrCF3 as a function of excitation wavelength, separately exciting either $S_{1}$ or $S_{2}$. For such a separate excitation of $S_{1}$, a large energy gap $\Delta E\left(S_{1}, S_{2}\right)$ is helpful. In the case of DMABN, $\Delta E\left(S_{1}, S_{2}\right)$ decreases with solvent polarity, in such a way that in $\mathrm{MeCN}$ the $S_{1}$ absorption band has merged with the main $S_{2}$ absorption band, as discussed above (Fig. 1). This condition restricts the experiments with DMABN to solvents of medium polarity such as THF, in which $\Delta E\left(S_{1}, S_{2}\right)$ is larger than in MeCN. The excitation wavelength dependence of the ICT reaction was therefore also investigated with $\mathrm{DPrCF} 3$, a molecule with a considerably larger $\Delta E\left(S_{1}, S_{2}\right)$ gap than DMABN (Fig. 4, Tables I and II).

\subsection{Excitation wavelength dependence of the ICT/LE fluorescence quantum yield ratio for DMABN in tetrahydrofuran}

The absorption and fluorescence spectra of DMABN in THF at $25^{\circ} \mathrm{C}$ are shown in Fig. 8. The overall fluorescence spectrum in Fig. 8a is separated into

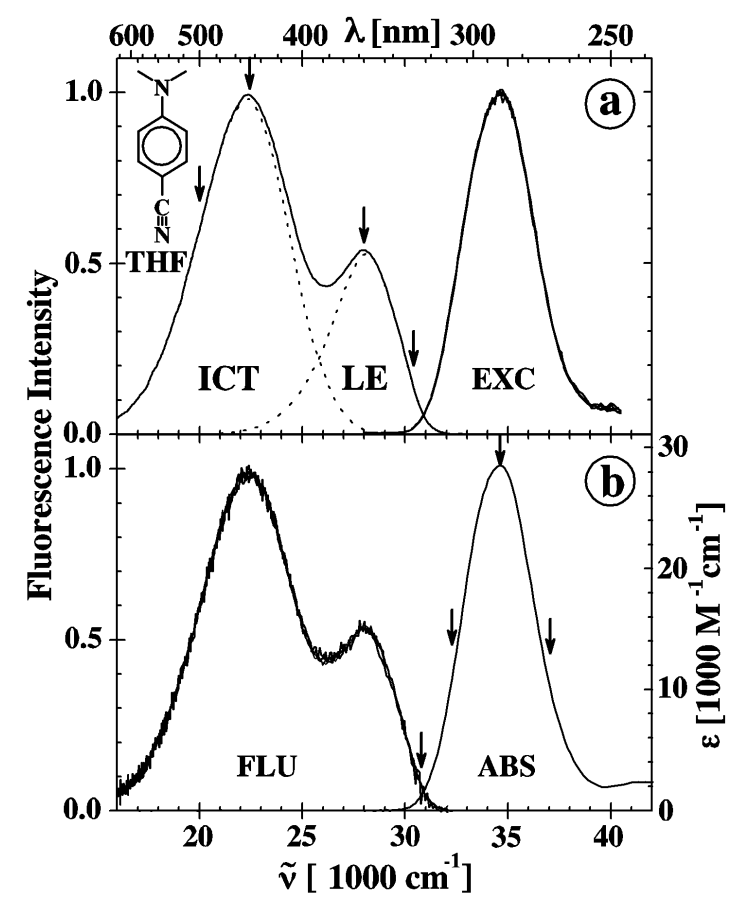

Fig. 8. (a) Excitation (EXC) and fluorescence spectra of DMABN in THF at $25^{\circ} \mathrm{C}$. The same excitation spectrum (EXC) is obtained for four different emission wavelengths (arrows) over the absorption spectrum (ABS). (b) The same fluorescence spectrum and ICT/LE fluorescence quantum yield ratio, see (a), is found at four different excitation wavelengths (arrows). The overall fluorescence spectrum in (a) has been separated into the contributions from the LE and ICT emissions. The fluorescence spectrum of MABN is adopted for the LE emission. 
the LE and ICT contributions. By monitoring two LE and two ICT emission wavelengths, the same excitation spectrum is obtained (Fig. 8a). These excitation spectra are similar to the absorption spectrum of DMABN in Fig. 8b. Likewise, by varying the excitation wavelength over the $S_{1} / S_{2}$ absorption band (Fig. 8b), the same overall fluorescence spectrum is found. These results indicate that the fluorescence quantum yield ratio $\Phi^{\prime}(\mathrm{ICT}) / \Phi(\mathrm{LE})$ is independent of excitation wavelength, even when $S_{2}$ is not excited.

\subsection{Excitation wavelength dependence of the ICT/LE fluorescence quantum yield ratio for DPrCF3 in acetonitrile}

The absorption and fluorescence spectra of DPrCF3 in $\mathrm{MeCN}$ at $25^{\circ} \mathrm{C}$ are shown in Fig. 9. For two LE and two ICT emission wavelengths, the same excitation spectrum is obtained (Fig. 8a), which is practically identical to the absorption spectrum of DPrCF3 in Fig. 9b. Similarly, by varying the excitation wavelength

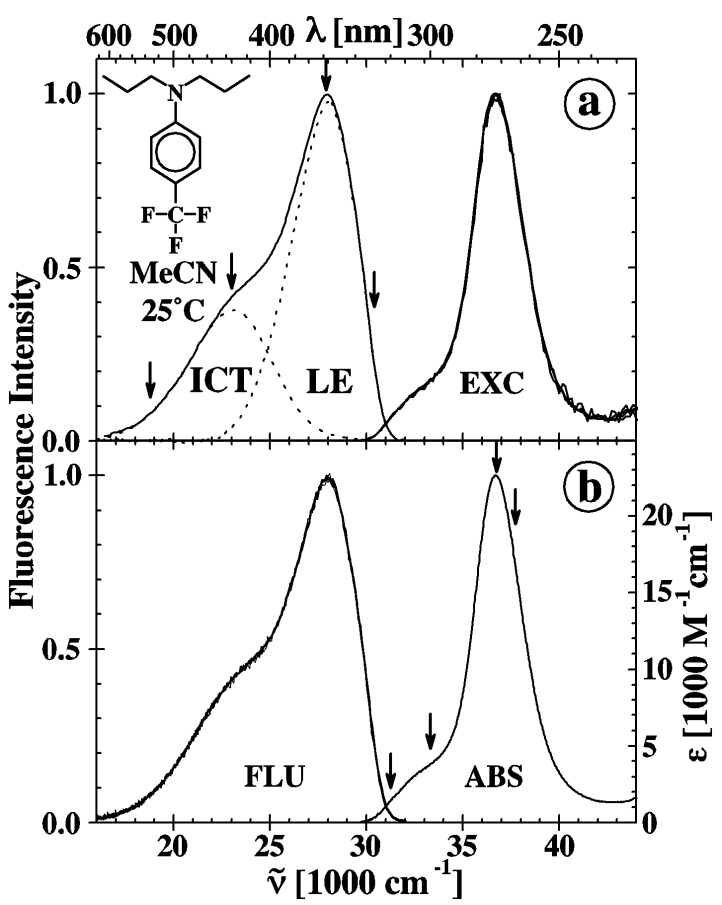

Fig. 9. (a) Excitation (EXC) and fluorescence spectra of DPrCF3 in $\mathrm{MeCN}$ at $25^{\circ} \mathrm{C}$. The same excitation spectrum (EXC), similar to the absorption spectrum (ABS) in (b), is obtained for four different emission wavelengths (arrows). (b) The same fluorescence spectrum and ICT/LE fluorescence quantum yield ratio, see (a), is found at four different excitation wavelengths (arrows). The overall fluorescence spectrum in (a) has been separated into the contributions from the LE and ICT emissions. The fluorescence spectrum of $\mathrm{N}$ - $n$-propyl-4-(trifluoromethyl)aniline (PrCF3) is adopted for the LE emission. 
over the absorption band (Fig. 9b), with two excitations into the $S_{1}$ absorption band, the same overall fluorescence spectrum is found. These results indicate that for DPrCF3 in MeCN, similar to what was found for DMABN in THF (Fig. 8), the fluorescence quantum yield ratio $\Phi^{\prime}(\mathrm{ICT}) / \Phi(\mathrm{LE})$ is independent of excitation wavelength, even when only $S_{1}$ is excited.

\subsection{Independence of excitation wavelength. LE as the ICT precursor in an adiabatic ICT reaction pathway for DPrCF3 and DMABN}

The independence of $\Phi^{\prime}(\mathrm{ICT}) / \Phi(\mathrm{LE})$ on excitation wavelength found for DMABN in THF and for DPrCF3 in MeCN indicates that for both D/A molecules the ICT reaction follows an adiabatic reaction pathway, with LE as the precursor of the ICT state. This conclusion supports earlier findings based on time-resolved fluorescence spectra of DMABN and NTC6, which showed that just after excitation the fluorescence spectrum consists of a single LE band, whereas the ICT emission grows in with time [10,23,32]. The relatively large energy separation between the $S_{1}$ and $S_{2}$ states in the case of DPrCF3 makes this molecule particularly suitable for investigations of excitation wavelength dependence of ICT reactions.

\section{Conclusions}

With a series of N,N-di- $n$-alkyl-4-(trifluoromethyl)anilines (DXCF3) with $\mathrm{X}=\mathrm{M}$ (methyl), $\mathrm{E}$ (ethyl), $\mathrm{Pr}$ ( $n$-propyl) and $\mathrm{Pe}(n$-pentyl), an ICT reaction takes place in the polar solvent $\mathrm{MeCN}$, but not in less polar solvents $n$-hexane and diethyl ether. The ICT reaction efficiency for DMCF3 in MeCN at $25^{\circ} \mathrm{C}$ is much smaller than that of DMABN in this solvent, as seen from the $\Phi^{\prime}(\mathrm{ICT}) / \Phi(\mathrm{LE})$ fluorescence quantum yield ratios: 0.06 for DMCF3 and 39.5 for DMABN. This difference is caused by the considerably larger energy gap $\Delta E\left(S_{1}, S_{2}\right)$ of DMCF3 $\left(5750 \mathrm{~cm}^{-1}\right)$ as compared with that of DMABN $\left(3130 \mathrm{~cm}^{-1}\right)$, in accordance with the requirements of the PICT model. For DXCF3s in MeCN, $\Phi^{\prime}(\mathrm{ICT}) / \Phi(\mathrm{LE})$ becomes larger with increasing amino-alkyl chain length: from 0.06 for DMCF3 to 0.64 for DPeCF3. This increase in ICT reaction efficiency is accompanied by a small but significant decrease in $\Delta E\left(S_{1}, S_{2}\right)$, from $5750 \mathrm{~cm}^{-1}$ for DMCF3 to $5370 \mathrm{~cm}^{-1}$ for DPeCF3.

The relatively large $\Delta E\left(S_{1}, S_{2}\right)$ gap of the DXCF3s makes these molecules suitable for an investigation of the excitation wavelength dependence of the ICT reaction. For DPrCF3 in MeCN it was found that $\Phi^{\prime}(\mathrm{ICT}) / \Phi(\mathrm{LE})$ is the same for excitation in the $S_{1}$ or in the $S_{2}$ manifold. A similar result was obtained with DMABN in THF. From these experiments it is concluded that for DPrCF3 and DMABN the ICT reaction follows an adiabatic pathway. After excitation to a Franck-Condon $S_{n}(\mathrm{FC})$ state, the A/D molecules first undergo ultrafast internal conversion to the relaxed $\operatorname{LE}\left(S_{1}\right)$ state, from which precursor the reaction then proceeds to the ICT state. Experimental evidence for a nonadiabatic ICT reaction pathway, going directly from $S_{2}(\mathrm{FC})$ via an $S_{2} / S_{1}$ conical intersection to LE and ICT, is not found. Such a reaction pathway is expected to result in an enhancement 
of the ICT population, i.e., in an increase in $\Phi^{\prime}(\mathrm{ICT}) / \Phi(\mathrm{LE})$, as compared with the adiabatic LE $\rightarrow$ ICT reaction. When the ICT state of DMABN or NMC6 would be directly prepared by passing through the $S_{2} / S_{1}$ CI, the fluorescence in the gas phase and in alkane solvents should contain an ICT emission, which is not observed in the experimental spectra.

\section{Acknowledgments}

Many thanks are due to Mr. Jürgen Bienert for carrying out the HPLC purifications and to Mr. Wilfried Bosch and Mr. Helmut Lesche for technical support. Dr. Attila Demeter and Dr. Sergey Druzhinin are thanked for their comments on the manuscript.

\section{References}

[1] E. Lippert, W. Lüder, H. Boos, in: Advances in Molecular Spectroscopy, European Conf. on Molecular Spectroscopy, Bologna (Italy) 1959, Ed. A. Mangini, Pergamon Press, Oxford, UK 1962, p. 443.

[2] E. Lippert, W. Lüder, F. Moll, W. Nägele, H. Boos, H. Prigge, I. Seibold-Blankenstein, Angew. Chem. 73, 695 (1961).

[3] Z.R. Grabowski, K. Rotkiewicz, W. Rettig, Chem. Rev. 103, 3899 (2003).

[4] K.A. Zachariasse, S.I. Druzhinin, W. Bosch, R. Machinek, J. Am. Chem. Soc. 126, 1705 (2004).

[5] T. Yoshihara, S.I. Druzhinin, K.A. Zachariasse, J. Am. Chem. Soc. 126, 8535 (2004)

[6] S.E. Braslavsky, Pure Appl. Chem. 79, 293 (2007).

[7] K. Rotkiewicz, Z.R. Grabowski, A. Krówczyński, W. Kühnle, J. Lumin. 12/13, 877 (1976)

[8] K.A. Zachariasse, M. Grobys, Th. von der Haar, A. Hebecker, Yu.V. Il'ichev, Y.-B. Jiang, O. Morawski, W. Kühnle, J. Photochem. Photobiol., A: Chem. 102, 59 (1996). Erratum, ibid. 115, 259 (1998).

[9] K.A. Zachariasse, M. Grobys, Th. von der Haar, A. Hebecker, Yu.V. Il'ichev, O. Morawski, I. Rückert, W. Kühnle, J. Photochem. Photobiol., A: Chem. 105, 373 (1997).

[10] S.I. Druzhinin, N.P. Ernsting, S.A. Kovalenko, L.P. Lustres, T.A. Senyushkina, K.A. Zachariasse, J. Phys. Chem. A 110, 2955 (2006).

[11] W. Fuß, K.K. Pushpa, W. Rettig, W.E. Schmid, S.A. Trushin, Photochem. Photobiol. Sci. 1, 255 (2002).

[12] W. Fuß, W.E. Schmid, K.K. Pushpa, S.A. Trushin, T. Yatsuhashi, Phys. Chem. Chem. Phys. 9, 1151 (2007).

[13] A. Köhn, C. Hättig, J. Am. Chem. Soc. 126, 7399 (2004).

[14] I. Gómez, M. Reguero, M. Boggio-Pasqua, M.A. Robb, J. Am. Chem. Soc. 127, 7119 (2005).

[15] I. Gómez, Y. Mercier, M. Reguero, J. Phys. Chem. A 110, 11455 (2006). 
[16] C. Hättig, A. Hellweg, A. Köhn, J. Am. Chem. Soc. 128, 15672 (2006).

[17] K.A. Zachariasse, M. Grobys, E. Tauer, Chem. Phys. Lett. 274, 372 (1997).

[18] C. Bulliard, M. Allan, G. Wirtz, E. Haselbach, K.A. Zachariasse, N. Detzer, S. Grimme, J. Phys. Chem. A 103, 7766 (1999).

[19] T.P. Carsey, G.L. Findley, S.P. McGlynn, J. Am. Chem. Soc. 101, 4502 (1979).

[20] A. Demeter, K.A. Zachariasse, Chem. Phys. Lett. 380, 699 (2003).

[21] J.A. Zablocki, J.G. Rico, R.B. Garland, T.E. Rogers, K. Williams, L.A. Schretzmann, S.A. Rao, P.R. Bovy, F.S. Tjoeng, R.J. Lindmark, J. Med. Chem. 38, 2378 (1995).

[22] J.N. Demas, G.A. Crosby, J. Phys. Chem. 75, 991 (1971).

[23] S.I. Druzhinin, A. Demeter, V.A. Galievsky, T. Yoshihara, K.A. Zachariasse, J. Phys. Chem. A 107, 8075 (2003).

[24] M. Grobys, K.A. Zachariasse, J. Information Recording 24, 405 (1998).

[25] J. Herbich, K. Rotkiewicz, J. Waluk, B. Andresen, E.W. Thulstrup, Chem. Phys. 138, 105 (1989).

[26] R.O. Loutfy, Can J. Chem. 54, 1454 (1976).

[27] Yu.V. Il'ichev, W. Kühnle, K.A. Zachariasse, J. Phys. Chem. A 102, 5670 (1998).

[28] B. Stevens, M.I. Ban, Trans. Faraday Soc. 60, 1515 (1964).

[29] J.B. Birks, Photophysics of Aromatic Molecules, Wiley, London 1970.

[30] K.A. Zachariasse, Trends in Photochemistry and Photobiology 3, 211 (1994).

[31] M. Kasha, Discuss. Faraday Soc. 9, 14 (1950).

[32] S.I. Druzhinin, S.A. Kovalenko, T.A. Senyushkina, K.A. Zachariasse, J. Phys. Chem. A, accepted for publication.

[33] S.I. Druzhinin, I. Dix, P. Mayer, M. Noltemeyer, K.A. Zachariasse, in preparation. 